\title{
Effect of capping rate on InAs/GaAs quantum dot solar cells
}

\author{
L. Stanojevića ${ }^{a}$ A. Gonzalo ${ }^{a}$, A. D. Utrilla ${ }^{a}$, D. F. Reyes ${ }^{b}$, V. Braza ${ }^{b}$, D. González ${ }^{b}$, D. Fuertes \\ Marrón $^{\mathrm{c}}$, A. Hierro ${ }^{\mathrm{a}}$, J.M. Ulloa*a \\ ${ }^{a}$ Institute for Systems based on Optoelectronics and Microtechnology (ISOM), Universidad \\ Politécnica de Madrid, Avda. Complutense 30, 28040 Madrid, Spain; \\ ${ }^{b}$ Departamento de Ciencia de los Materiales e IM y QI, Universidad de Cádiz, 11510 Puerto Real, \\ Cádiz, Spain \\ 'Instituto de Energía Solar (IES), Universidad Politécnica de Madrid, Avda. Complutense 30, 28040 \\ Madrid, Spain
}

\begin{abstract}
The unavoidable presence of the wetting layer (WL) in Stranski-Krastanov quantum dots (QD) has typically a negative impact on the performance of QD solar cells. In this work, a simple method to engineer the WL of InAs/GaAs QD solar cells is investigated. In particular, we show that covering the QDs at high GaAs capping rates reduces In-Ga intermixing and, therefore, In redistribution from the QDs to the WL. This results not only in larger QDs, but also in thinner WLs, with larger quantum confinement energies and reduced potential barriers for electrons and holes. Carrier trapping by the WLs and subsequent recombination is therefore reduced, resulting in larger photocurrent values of the QD solar cells under short circuit conditions.
\end{abstract}

Keywords: quantum dots, wetting layer, quantum dot solar cell, capping rate, carrier trapping

\section{INTRODUCTION}

Different constraints of bulk single-junction single bandgap semiconductor solar cells led to the development of various theoretical and practical concepts and strategies, mostly known under one common name - third generation solar cells, such as multi-junction solar cells (MJSCs), intermediate band solar cells (IBSCs) etc. Quantum dots (QD) are very attractive in this field, not only as a strategy to overcome the Shockley-Queisser efficiency limit through novel concepts such as IBSCs, but also as a way to extend the effective bandgap of single or multi-junction (sub)cells to the near infrared [1], [2]. Nevertheless, the efficiency of semiconductor QD solar cells (QDSC) has hardly reached the level of conversion efficiency of the control solar cells without QDs so far. This is due to several problems, in which the wetting layer (WL) may have a relevant but relatively unexplored role.

InAs/GaAs QD structures are usually grown by the Stanski-Krastanov (SK) epitaxial method. It means that WLs, thin quantum well-like (2D) structures, are formed first, followed by QD formation on top of them. The subsequent necessary process of capping the QDs is well known as a cause for their partial decomposition due to a strong In-Ga intermixing. This results in migration of In atoms from quantum dots towards the wetting layer leading to smaller QDs and thicker WLs after capping with GaAs [3]-[9]. Therefore, depending on the initial QD size, the actual WL in the QDSC can be quite thick, which should affect device performance. Since carrier transport through the structure is transversal to the wetting layers (i.e. parallel to the growth direction), it can be hindered by carrier trapping in the WLs, followed by recombination in the WL itself or injection from the WL to the QDs and radiative recombination there. Therefore, different strategies are being investigated nowadays in the field of QDSCs with the aim of reducing the impact of the WL in solar cell performance, such as inserting thin AlAs [10], [11] or GaAsSb [12] capping layers. This strategies, as well as using other capping materials such as $\mathrm{GaAsN}(\mathrm{Sb})$ [13], however, inevitably lead to changes in the band structure that may have other undesirable consequences [14].

In this work, we investigate a very simple method of reducing the impact of the WL in QDSCs. We show that the WL thickness is reduced by growing the first monolayers (ML) of the GaAs capping layer at high growth rates, due to a reduction or suppression of the decomposition process. We analyze the photocurrent characteristics of InAs/GaAs QDSCs with different capping rates.

*jmulloa@isom.upm.es 


\section{EXPERIMENTAL DETAILS}

\subsection{Growth details}

All samples were grown by solid source molecular beam epitaxy on $n+(100)$ GaAs substrates under As $_{4}$ overpressure. One sample was grown for transmission electron microscopy (TEM) analysis and it contains three QD layers grown at $450{ }^{\circ} \mathrm{C}$ by depositing $2.7 \mathrm{MLs}$ of InAs at $\sim 0.04 \mathrm{ML} / \mathrm{s}$. Each of the three QD layers was subsequently capped at $470{ }^{\circ} \mathrm{C}$ by a $30 \mathrm{ML} /$ thick GaAs capping layer grown at capping rates of $0.5 \mathrm{ML} / \mathrm{s}, 1.0 \mathrm{ML} / \mathrm{s}$ and $2.0 \mathrm{ML} / \mathrm{s}$, respectively. The QD layers were separated from each other by $50 \mathrm{~nm}$-thick GaAs spacer layers grown at $580^{\circ} \mathrm{C}$ and $1.0 \mathrm{ML} / \mathrm{s}$. Finally, a 200 nm-thick intrinsic GaAs layer at $1.0 \mathrm{ML} / \mathrm{s}$ and $580^{\circ} \mathrm{C}$ was deposited, on top of which a fourth layer of uncapped QDs was grown for reference. The series of p-i-n junction solar cells consists of two samples made for photocurrent spectroscopy analysis. In this series of samples the active region consists of 10 InAs QD layers grown under the same conditions than those in the previous sample. The QD layers in the two samples were covered by a $30 \mathrm{ML}$-thick GaAs capping layer grown at $1.0 \mathrm{ML} / \mathrm{s}$ and $2.0 \mathrm{ML} / \mathrm{s}$, respectively. The 10 QD layers in the solar cells were separated by $35 \mathrm{~nm}$-thick GaAs spacer layers. The whole active region was sandwiched between two $200 \mathrm{~nm}$-thick intrinsic GaAs regions. This structure was grown on top of a $500 \mathrm{~nm}$-thick n-GaAs base buffer layer grown on the n-substrate and covered by a 350/150 nm-thick p+/p++ GaAs layer (Fig. 1).

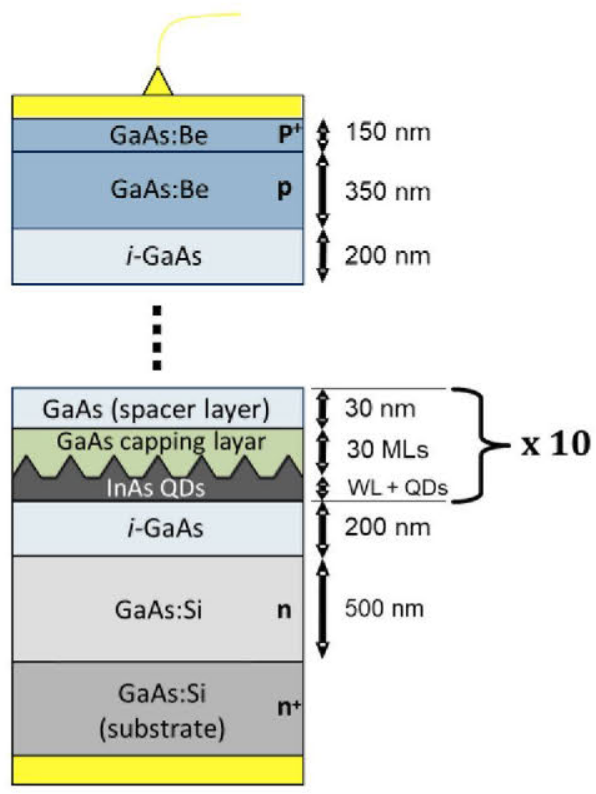

Figure 1. Sketch of the p-i-n devices containing 10 InAs QD layers capped with $30 \mathrm{ML}$-thick GaAs capping layers with different growth rates and separated by $35 \mathrm{~nm}$-thick spacer layers.

\subsection{Structural characterization, device processing and optoelectronic characterization}

The structural properties were analysed by conventional TEM techniques, using a JEOL JEM-2100 LaB6 microscope working at $200 \mathrm{kV}$. High resolution TEM images were post processed to obtain strain maps by using geometrical phase analysis (GPA) [15].

$200 \mu \mathrm{m}$-diameter cylindrical mesa-etched devices were processed through standard fabrication techniques using $\mathrm{AuGe} / \mathrm{Au}$ and $\mathrm{Au} / \mathrm{AuZn} / \mathrm{Au}$ for the $\mathrm{n}$ - and p-type contacts, respectively. Photocurrent spectroscopy was carried out using a Keithley 230 voltage source and a Keithley 617 electrometer, using light from a quartz halogen lamp dispersed through a $0.34 \mathrm{~m}$ monochromator. 


\section{RESULTS AND DISCUSSION}

\subsection{Effect of capping rate on WL thickness}

Fig. 2(a) shows g002 dark-field TEM images of a representative area of each of the layers of the sample designed for TEM, taken in the $\left[\begin{array}{lll}1 & 1 & 0\end{array}\right]$ pole axis. The QD height increases clearly with the capping rate, becoming progressively similar to that of the uncapped QDs. For a more detailed evaluation, especially having in mind the dispersion in the QD size and shape, a statistical analysis on the QD dimensions was performed by measuring height and diameter of more than 60 QDs from each layer. No significant difference in the QD base diameter was found. This dimension remains within the narrow range of $21.0 \pm 0.5 \mathrm{~nm}$ for each sample. On the other hand, the QD height shows a remarkable behavior exhibiting a linear dependence of the QD height with respect to the capping rate (see Fig. 2(b)). This means that we are progressively reducing QD decomposition at higher capping rates. Moreover, the height of the QDs covered at $2 \mathrm{ML} / \mathrm{s}$ is the same than in the uncapped QDs. This demonstrates that at the capping rate of $\sim 2.0 \mathrm{ML} / \mathrm{s}$ the dissolution process of buried QDs is completely blocked and the QD height is preserved as it was before capping. However, it should be noted that the QD height dispersion increases with the capping rate (the error bar in Fig. 2(b) represents the standard deviation). Interestingly, by suppressing the decomposition, the QD size dispersion increases, and vice versa, the decomposition process leads to better QD size uniformity. The possibility of controllably tuning the QD height, and therefore the QD electronic structure and optical properties, by this simple method could be interesting for many applications.

a)

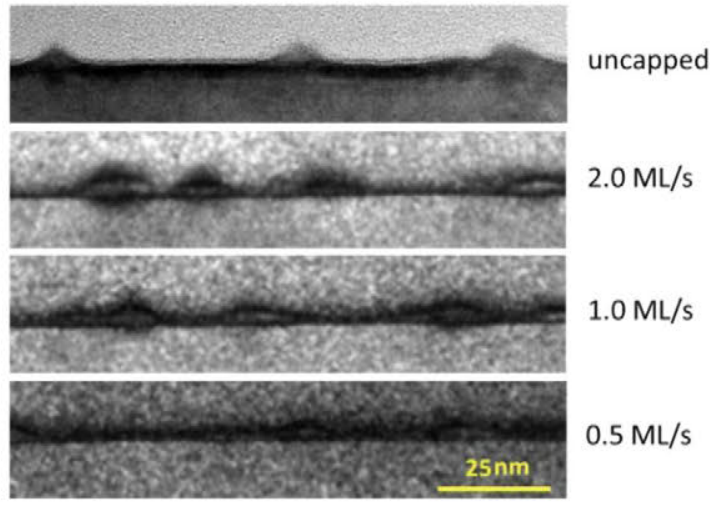

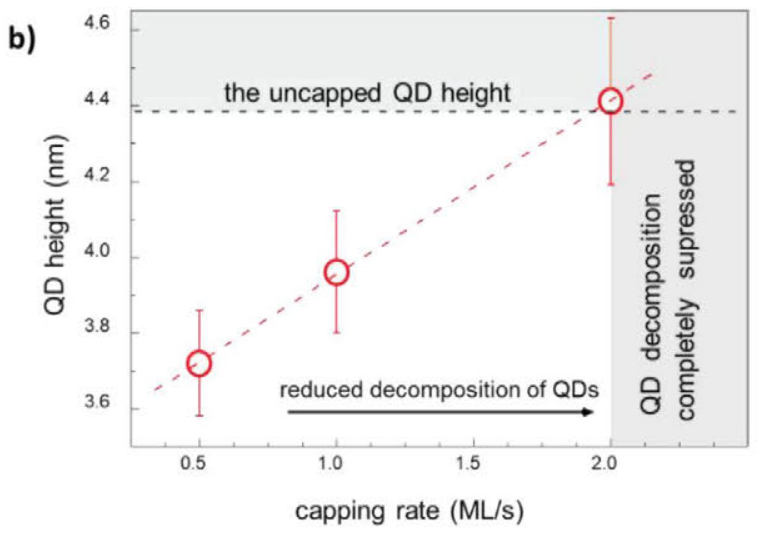

Figure 2. (a) g200 dark-field TEM images of the sample with QD layers capped at different rates and an uncapped QD layer on the surface. (b) Average QD height as a function of the capping rate in the sample studied by TEM. The black dashed line represents the uncapped QD height, indicating a completely suppressed decomposition process. The red dashed line is a linear fit to the data.

In principle, the reduced QD decomposition should result in a thinner WL. Fig. 3(a) shows the In content maps of the three layers capped at different capping rates obtained from HR-TEM images by using the thin foil approach in the GPA methodology [15]. The GPA images display a representative QD and WL region for each capping rate. Fig. 3(b) displays averaged profiles of In distribution along the growth direction obtained from the GPA maps. There is a clear reduction of the In content in the WL when the capping rate is increased. The WL capped at the highest growth rate $(2 \mathrm{ML} / \mathrm{s})$ has the smallest thickness (the In content curve is narrower and the maximum of the curve is the lowest of all three samples). Since for this growth rate the decomposition process is completely suppressed, this should be the thinnest WL layer achievable with our approach. The effect of the GaAs capping rate on the WL morphology has been recently confirmed by other techniques [8], [15]. A thinner WL should result in larger confinement energy and, therefore, reduced potential barriers for electrons and holes. This could facilitate carrier escape from the WL and reduce recombination. 
a)
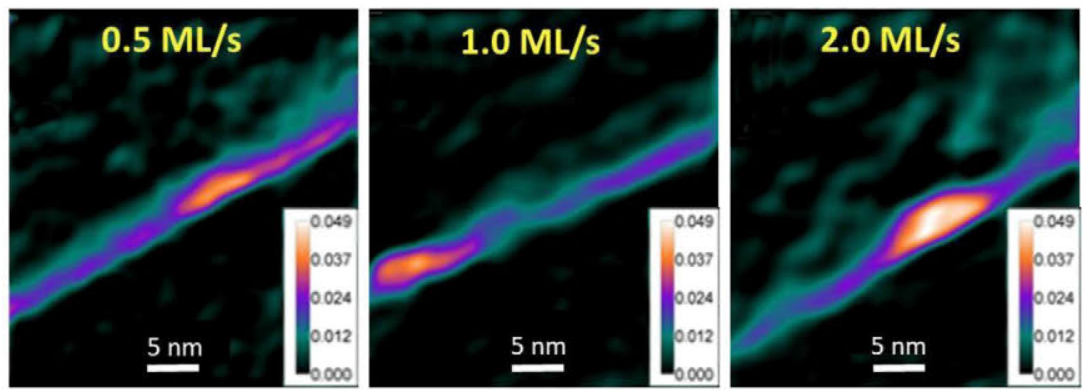

b)

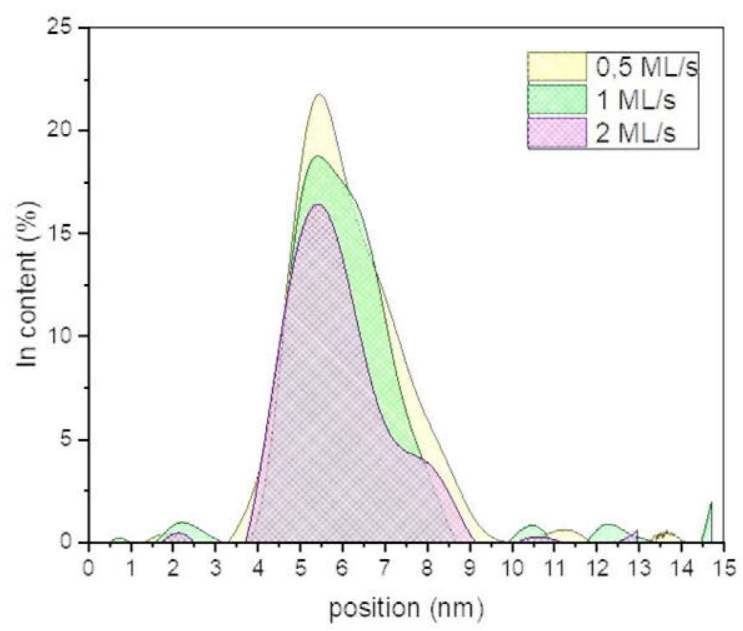

Figure 3. (a) In content maps of the layers capped at different rates using the thin foil approach in the GPA methodology [15]. (b) Mean In content profile of the WL in the growth direction obtained from GPA maps in the three layers.

\subsection{Effect of capping rate on photocurrent}

The modified WL morphology should in principle affect solar cell performance. In particular, reduced trapping of photogenerated carriers would be expected in thinner WLs, i.e., in high capping rates QDs. In order to investigate this, two QDSCs with 1 and $2 \mathrm{ML} / \mathrm{s}$ capping rates were analyzed. Having in mind that $1 \mathrm{ML} / \mathrm{s}$ is the standard capping rate and the clear correlation between the capping rate and the WL thickness shown before, the sample with QDs capped at $2 \mathrm{ML} / \mathrm{s}$ is expected to be far less affected by carrier trapping in WLs (and QDs). Fig. 4 displays the room temperature photocurrent spectra at $0 \mathrm{~V}$ of the two samples $(1 \mathrm{ML} / \mathrm{s}$ and $2 \mathrm{ML} / \mathrm{s})$. The photocurrent generated at energies lower than the GaAs bandgap is due to the absorption of nanostructures (WLs and QDs). As it can be seen in the figure, there is a clearly visible red shift (shift towards longer wavelengths / lower energies) in the region that corresponds to the QD ground state absorption edge (the range around $1.0-1.1 \mathrm{eV}$ ) when the capping rate is increased. This is in agreement with the increased $\mathrm{QD}$ height at high capping rates. The QDs capped at $2 \mathrm{ML} / \mathrm{s}$ absorb a broader region of the infrared spectrum. On the other hand, there is a blue shift (shift towards shorter wavelengths / higher energies) in the photocurrent absorption edge of the WL (approximately in the range of $1.3-1.4 \mathrm{eV}$ ). This is the result of a thinner WL, with larger quantum confinement energy and ground state transition energy. Remarkably, the photocurrent generated at GaAs (above $1.42 \mathrm{eV}$ ) is larger in the high capping rate solar cell (see inset of Fig. 4). This means that, indeed, trapping of carriers photogenerated at GaAs is reduced in that sample, likely due to the thinner WL. Overall, the integrated photocurrent under short circuit conditions is larger in the $2 \mathrm{ML} / \mathrm{s}$ QDSC, increasing by a 3,5\%. This should in principle result in an enhanced short circuit current in the $2 \mathrm{ML} /$ solar cell. A larger open circuit voltage would be also in principle expected since trapping and recombination are reduced for thinner WLs. Nevertheless, this is still subject of ongoing investigation. 


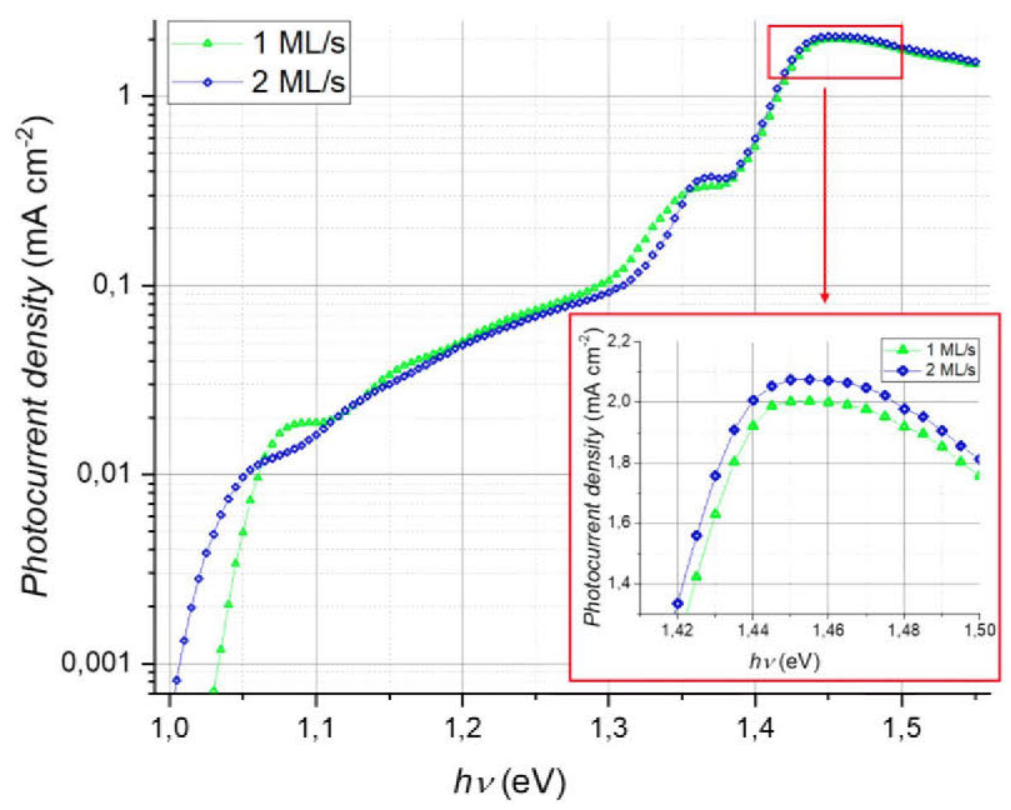

Figure 4. Photocurrent spectra of two QDSCs, each containing 10 InAs QD layers grown under the same conditions, capped with GaAs at two different growth rates: $1 \mathrm{ML} / \mathrm{s}$, and $2 \mathrm{ML} / \mathrm{s}$.

\section{CONCLUSION}

We have presented a comparative study of the structural properties of InAs/GaAs QDs and WLs covered with GaAs at different growth rates. This is a recently proposed straightforward method to tune size and shape of QDs, and therefore their optical properties. We applied this method to QDSCs, in order to obtain thinner WLs and mitigate its impact on QDSC performance. It is confirmed that, by increasing the capping rate to $2 \mathrm{ML} / \mathrm{s}$, the QD absorption edge is shifted towards lower photon energies, whereas the $\mathrm{WL}$ absorption edge is shifted towards higher photon energies. This results also in an enhancement of the photocurrent under short circuit conditions, which is explained in terms of reduced carrier trapping in the WL, although that phenomenon still has to be investigated in detail.

\section{ACKNOWLEDGEMENTS}

We acknowledge funding from the Spanish MINECO through project MAT2016-77491-C2-1-R and from the Comunidad de Madrid through project S2013/MAE-2780. We also would like to thank support from the EU through COST Action MP1406.

\section{REFERENCES}

[1] S. M. Hubbard, R. Raffaelle, S. Bailey, R. Raffaelle, and S. Bailey, "Quantum Dot Solar Cells" in Nanotechnology for Photovoltaics, 279-322, Ed. Taylor and Francis Group (2010)

[2] A. Martí, C. R. Stanley, and A. Luque, "Intermediate Band Solar Cells (IBSC) Using Nanotechnology," in Nanostructured Materials for Solar Energy Conversion, 539-566, Ed. Elsevier (2006)

[3] J. M. García, G. Medeiros-Ribeiro, K. Schmidt, T. Ngo, J. L. Feng, A. Lorke, J. Kotthaus, and P. M. Petroff, "Intermixing and shape changes during the formation of InAs self-assembled quantum dots", Appl. Phys. Lett. 71, 2014-2016 (1997). 
[4] G. D. Lian, J. Yuan, L. M. Brown, G. H. Kim, and D. A. Ritchie, "Modification of InAs quantum dot structure by the growth of the capping layer", Appl. Phys. Lett. 73, 49-51 (1998).

[5] G. Costantini, A. Rastelli, C. Manzano, P. Acosta-Diaz, R. Songmuang, G. Katsaros, O. G. Schmidt, and K. Kern, "Interplay between Thermodynamics and Kinetics in the Capping of InAs/GaAs(001) Quantum Dots", Physical Review Letters 96(22), 226106:1-226106:4 (2006).

[6] J. M. Ulloa, R. Gargallo-Caballero, M. Bozkurt, M. del Moral, A. Guzmán, P. M. Koenraad, and A. Hierro, "GaAsSb-capped InAs quantum dots: From enlarged quantum dot height to alloy fluctuations," Phys. Rev. B, 81(16), 165305:1-165305:7 (2010).

[7] Q. Gong, P. Offermans, R. Nötzel, P.M. Koenraad, J.H. Wolter, Capping process of InAs/GaAs quantum dots studied by cross-sectional scanning tunneling microscopy, Appl. Phys. Lett. 85, 5697-5699 (2004).

[8] D. González, D.F. Reyes, A.D. Utrilla, T. Ben, V. Braza, A. Guzman, A. Hierro, J.M. Ulloa, "General route for the decomposition of InAs quantum dots during the capping process", Nanotechnology 27, 125703:1-125703:8 (2016)

[9] A. D. Utrilla et al., "Size and shape tunability of self-assembled InAs/GaAs nanostructures through the capping rate," Appl. Surf. Sci., 444, 260-266 (2018).

[10] D. Hu, C. C. McPheeters, E. T. Yu, and D. M. Schaadt, "Improvement of performance of InAs quantum dot solar cell by inserting thin AlAs layers," Nanoscale Res. Lett., 6(1), 83 (2011).

[11] F. K. Tutu, P. Lam, J. Wu, N. Miyashita, Y. Okada, K.-H. Lee, N. J. Ekins-Daukes, J. Wilson, and H. Liu, "InAs/GaAs quantum dot solar cell with an AlAs cap layer", Appl. Phys. Lett. 102 (16), 163907 (2013).

[12] A.D. Utrilla, D.F. Reyes, J.M. Llorens, I. Artacho, T. Ben, D. González, Ž.Gačević, A. Kurtz, A. Guzman, A. Hierro, J.M. Ulloa, "Thin GaAsSb capping layers for improved performance of InAs/GaAs quantum dot solar cells," Sol. Energy Mater. Sol. Cells 159, 282-289 (2017).

[13] J.G. Keizer, J.M. Ulloa, A.D. Utrilla, P.M. Koenraad, InAs quantum dot morphology after capping with In, N, Sb alloyed thin films, Appl. Phys. Lett. 104, 053116:1-053116:5 (2014)

[14] A.D.Utrilla, J.M.Ulloa, Ž. Gačević, D.F.Reyes, I.Artacho, T.Ben, D.González, A.Hierro, A.Guzman, "Impact of alloyed capping layers on the performance of InAs quantum dot solar cells", Sol. Energy Mater. Sol. Cells 144, 128-135 (2016).

[15] D González, V Braza, A D Utrilla, A Gonzalo, D F Reyes, T Ben, A Guzman, A Hierro and J M Ulloa, "Quantitative analysis of the interplay between InAs quantum dots and wetting layer during the GaAs capping process," Nanotechnology 28, 425702 (2017). 